Published in final edited form as:

Ann Epidemiol. 2018 February ; 28(2): 102-106.e1. doi:10.1016/j.annepidem.2017.12.003.

\title{
Diabetes-related Factors and Abdominal Aortic Aneurysm Events: the Atherosclerotic Risk in Communities (ARIC) Study
}

\author{
Yasuhiko Kubota, MD ${ }^{1}$, Aaron R. Folsom, MD, MPH${ }^{1}$, James S. Pankow, MPH, PhD ${ }^{1}$, Lynne \\ E. Wagenknecht, $\mathrm{DrPH}^{2}$, and Weihong Tang, MD, $\mathrm{PhD}^{1}$ \\ ${ }^{1}$ Division of Epidemiology and Community Health, School of Public Health, University of \\ Minnesota, Minneapolis, MN, USA \\ ${ }^{2}$ Department of Epidemiology and Prevention, Wake Forest School of Medicine, Winston Salem, \\ NC, USA
}

\section{Abstract}

Purpose-To test the hypothesis that diabetes-related factors [metabolic syndrome (MetS), glucose, insulin, and leptin] are inversely associated with abdominal aortic aneurysm (AAA) risk.

Methods-We followed 13,736 participants, aged 45-64, without prior AAA surgery at baseline (1987-1989), for AAA occurrence through 2011. Hazard ratios (HRs) and their 95\% confidence intervals (CIs) of AAA were calculated using Cox regression.

Results-During 275,054 person-years of follow-up, we identified 518 AAA events. Fasting serum glucose was associated inversely with AAA risk [HR (95\% CI) per 1 unit increment in $\log _{2}$ (glucose), 0.54 (0.36-0.80)], but fasting insulin was not associated with AAA. Plasma leptin was also associated inversely with AAA occurrence [HR $(95 \% \mathrm{CI})$ per 1 unit increment in $\log _{2}$ (leptin), 0.83 (0.71-0.98)]. Compared with individuals without MetS, those with MetS had increased risk of AAA [HR (95\% confidence interval CI): 1.24 (1.04-1.48)]. Among individuals with or without diabetes, the HRs increased monotonically with a greater number of non-glucose MetS components.

Conclusion-Diabetes, fasting glucose, and plasma leptin were inversely associated with risk of AAA. In contrast, the MetS was associated with increased risk of AAA, due to the influence of the non-glucose MetS components.

\section{Keywords}

diabetes; abdominal aortic aneurysm; leptin; metabolic syndrome

Correspondence to Yasuhiko Kubota, Division of Epidemiology and Community Health, School of Public Health, University of Minnesota, 1300 South 2nd Street, Minneapolis, MN 55454. Tel: 612-625-1016; Fax: 612-624-0315; kubot007@umn.edu.

DISCLOSURES

All authors have approved the final article.

CONFLICT OF INTEREST

The Authors have no conflict of interest to declare.

Publisher's Disclaimer: This is a PDF file of an unedited manuscript that has been accepted for publication. As a service to our customers we are providing this early version of the manuscript. The manuscript will undergo copyediting, typesetting, and review of the resulting proof before it is published in its final citable form. Please note that during the production process errors may be discovered which could affect the content, and all legal disclaimers that apply to the journal pertain. 


\section{INTRODUCTION}

Abdominal aortic aneurysm (AAA) is an important cause of death in Western countries, especially in old age $(1,2)$. Several risk factors for AAA have been identified, including atherosclerosis, old age, male sex, hypertension, dyslipidemia and smoking (1). In contrast, diabetes mellitus (DM), surprisingly, is inversely associated with AAA risk (3-5). In addition, a previous study suggested obesity might also be inversely associated with AAA (6) although this appears controversial $(7,8)$. Obesity is closely related to DM, and thus, other DM-related factors such as blood insulin concentrations or the metabolic syndrome (MetS) might also be inversely associated with AAA risk. To date, there is no prospective study investigating the association of these DM-related factors with AAA.

The Atherosclerosis Risk in Communities Study (ARIC) measured on its cohort several DM-related variables-MetS, fasting serum glucose and insulin, and plasma leptin (9)—and has identified incident, clinical AAAs through 2011. Therefore, the primary and secondary objectives of this study were to examine the association of MetS and those plasma biomarkers with AAA risk, respectively.

\section{MATERIALS AND METHODS}

\section{Study Design, Setting, and Population}

The ARIC Study recruited 15,792 mostly white or African American men and women aged 45-64 from 4 U.S. communities [Jackson, Mississippi (African Americans only); Washington County, Maryland; suburbs of Minneapolis, Minnesota; and Forsyth County, North Carolina] to a baseline examination between 1987 and 1989 (10). Major cardiovascular risk factors were measured through home interview and clinic examinations. This study was conducted according to the guidelines of the Helsinki Declaration.

In the present analyses, we excluded participants who at baseline in 1987-1989 reported prior AAA surgery or aortic angioplasty $(\mathrm{n}=11)$, had uncertain AAA status during follow-up $(\mathrm{n}=30)$, were non-white participants in Washington County or Minneapolis or non-white/ black participants in Forsyth County $(\mathrm{n}=48)$ in order to allow multivariable adjustment for race and study site (17), or were participants whose data on main exposures (MetS and fasting glucose and insulin) ( $n=927)$ or any other covariates $(n=1,013)$ were missing. After exclusions, 13,763 participants were included in the present analyses. For the leptin analysis, after identical exclusions, 701 participants were available. Assuming the sample size of our participants, the estimated proportion of AAA cases in reference group $\geq 0.01$, type I error $=0.05$, relative risk $=0.5$ (decreased risk) or 2.0 (increased risk) and $\geq 20$ years of followup, we obtained study power $\searrow 0.8$.

The institutional review boards of the collaborating universities approved the protocol, and ARIC obtained written informed consent from all participants. 


\section{Exposure and Covariates}

DM was defined as a fasting serum glucose $\geq 126 \mathrm{mg} / \mathrm{dl}$, non-fasting serum glucose $\geq 200$ $\mathrm{mg} / \mathrm{dl}$, a self-reported physician diagnosis of diabetes, or on treatment for diabetes mellitus in the past 2 weeks (11). MetS was defined by the presence of at least 3 of the following components: (i) central obesity; waist circumference $\geq 102 \mathrm{~cm}$ in men or $\geq 88 \mathrm{~cm}$ in women, (ii) low $\mathrm{HDL}$ cholesterol; plasma $\mathrm{HDL}<1.0 \mathrm{mmol} / \mathrm{L}$ in men and $<1.3 \mathrm{mmol} / \mathrm{L}$ in women or on lipid medication, (iii) hypertension; systolic blood pressure $\geq 130 \mathrm{~mm} \mathrm{Hg}$ or diastolic blood pressure $\geq 85 \mathrm{~mm} \mathrm{Hg}$ or on antihypertensive medication, (iv) hypertriglyceridemia; plasma triglycerides $\geq 1.7 \mathrm{mmol} / \mathrm{L}$ or on lipid medication, and (v) abnormal glucose metabolism; fasting serum glucose $\geq 100 \mathrm{mg} / \mathrm{dL}$ or on treatment for diabetes mellitus (12, 13). Glucose was measured by a hexokinase method on a Coulter DACOS (Coulter Instruments), and insulin was measured with a commercial radioimmunoassay (Cambridge Biomedical) (14). Based on our internal quality control materials, the interassay analytical standard deviation was $1.3 \mathrm{mg} / \mathrm{dL}$ (percent coefficient of variation, $1.6 \%$ ) at $79.3 \mathrm{mg} / \mathrm{dL}$ for glucose and $16.5 \mathrm{mU} / \mathrm{L}$ (percent coefficient of variation, 17\%) at $96.9 \mathrm{mU} / \mathrm{L}$ for insulin (14). Plasma leptin (reliability coefficient based on split specimens was 0.94 ) had been measured in duplicate by direct sandwich ELISA (Linco Research, St Charles, MI, USA) (9) in a previous nested case-coort study of incident coronary heart disease from ARIC visit 1 to December 31, 1993. The cohort reference group, the focus of the present leptin analysis, was a stratified random sample of participants free of baseline coronary heart disease in the ARIC cohort, with oversampling of participants with thin average carotid intima-media thickness measurements at baseline ( $<30$ th percentile) and different sampling fractions by age sex, and race (15).

We also included in analysis other risk factors (covariates) for AAA in ARIC $(8,16)$, including age, sex, race (white or African American), height $(\mathrm{cm})$, smoking status (current, former, or never), pack-years of smoking, plasma low-density lipoprotein (LDL) cholesterol, and history of peripheral artery disease.

\section{Identification of Abdominal Aortic Aneurysm}

ARIC identified incident clinical AAAs by several strategies $(8,16)$. During annual telephone calls, ARIC participants were asked about any interim hospitalizations and participants' deaths were identified. Surveillance of local hospitals was also conducted to identify additional hospitalizations or deaths. Moreover, participant identifiers were linked with fee-for-service Medicare data from the Centers for Medicare and Medicaid Services, to find additional hospital or outpatient AAA events for participants over 65 years. ARIC identified incident clinical AAAs as those with a hospital discharge diagnosis from any source, or two Medicare outpatient claims that occurred at least one week apart, with ICD-9$C M$ codes of 441.3 or 441.4 , or procedure codes of 38.44 or 39.71 , or death codes, ICD- 9 441.3 or 441.4 or $I C D-10$ code I71.3 or I71.4. AAAs based on procedure codes were required to be verified by diagnosis codes. Some of these clinical diagnoses would include asymptomatic AAAs that happened to be clinically documented. 


\section{Statistical Analysis}

SAS version 9.4 software (SAS Institute Inc., Cary, NC) was used for statistical analyses. All statistical tests were two-tailed and $P$ values $<0.05$ were regarded as significant.

We computed mean levels or percentages of potential AAA risk factors at baseline according to the presence or absence of DM. Person-years of follow-up were calculated from baseline to the first endpoint: AAA, death, loss to follow-up, or administrative censoring at December 31, 2011. Hazard ratios (HRs) and their 95\% confidence intervals (CIs) of clinical AAA were calculated after adjustment for other AAA risk factors using Cox proportional hazards model (for leptin analysis, stratified sampling weights using a weighted Cox proportional hazard models were used to account for sampling). The proportional hazards assumption in the Cox regression was tested using risk factor-by-time interactions and was not violated. Since we found no statistical interactions between sex or race and DM-related factors in relation to AAA risk, we pooled the analysis across sex and race. Plasma fasting glucose and insulin, and leptin were modeled using continuous variables, with $\log _{2}$-transformed values because of skewness.

For sensitivity analyses, we (i) further adjusted for abnormal glucose metabolism (fasting serum glucose $\geq 100 \mathrm{mg} / \mathrm{dL}$ or on treatment for diabetes mellitus) for the analysis of the associations between the number of MetS components and risk of AAA, and (ii) reran models for this analysis after changing the definition of the reference group from the number of MetS components, $0-1$ to 0 or $0-2$.

\section{RESULTS}

\section{Baseline Characteristics of Study Participants by Diabetes}

Compared with individuals without DM, those with DM tended to be older, male, African American, shorter in stature, and non-current smokers, have more pack-years of smoking, more hypertension and peripheral artery disease; and have a lower HDL cholesterol level and a higher LDL cholesterol level (Table 1). Prevalence or means of the DM-related factors of interest were higher in individuals with DM than those without DM.

\section{Associations between Diabetes-related Biomarkers and Risk of Abdominal Aortic Aneurysm}

During the 275,054 person-years of follow-up for the 13,763 participants, we identified 518 incident clinical AAA events. Individuals with DM had a lower risk of AAA, and this association remained significant even after adjusting for competing risks of death from underlying causes other than AAA (Supplemental Table). A fully adjusted model (Model 2) showed that fasting serum glucose and plasma leptin were inversely associated with AAA risk, but fasting serum insulin was not associated with AAA risk (Table 2). After excluding individuals with DM, fasting glucose showed no association with AAA, but leptin was still inversely associated with AAA. 


\section{Associations between Metabolic Syndrome and Risk of Abdominal Aortic Aneurysm}

In the final model (Table3, Model 2), compared with individuals without MetS, those with MetS had increased risk of AAA [HR 95\% CI: 1.24 (1.04-1.48)]. Table 4 shows the associations between the number of MetS components and risk of AAA in those with or without DM. For this analysis, abnormal glucose metabolism was not included in the number of MetS components because glucose and DM were inversely associated with AAA risk in contrast to the other components. The age-, sex-, and race-adjusted model (Model 1) showed that among in both those without or with DM, the HRs increased monotonically with a greater number of non-glucose MetS components. Yet, those with DM had a lower or similar risk of AAA, compared to those without DM, regardless of the number of nonglucose MetS components (central obesity, low HDL cholesterol, hypertension and hypertriglyceridemia) (Table 4). Further adjustment for other AAA risk factors showed similar associations. There was no significant interaction between the number of nonglucose MetS components and DM in relation to AAA risk, thus suggesting that nonglucose MetS components are associated similarly with AAA in those with versus without DM.

\section{Sensitivity Analyses}

Both sensitivity analyses mentioned in Methods produced similar results to main results (data not shown).

\section{DISCUSSION}

In this population-based prospective study, DM, leptin and fasting glucose were inversely associated with AAA risk, but fasting insulin was not. The inverse association between leptin and AAA appeared independent of DM. Participants with the MetS had an increased risk of AAA compared with normal participants, and participants with more non-glucose MetS components had a greater risk of AAA. In contrast, those with DM had a lower or similar risk of AAA than those without DM regardless of the number of MetS components.

One of our novel findings is the inverse association between leptin and AAA risk. Although elevated leptin, usually reflecting leptin resistance, is closely related to obesity (18), we previously showed no association between obesity and AAA (8). Several previous studies have suggested that elevated leptin predicts increased risk of DM as well as glucose intolerance (19-22). Thus, the association observed in this study might be related to those with elevated leptin being associated with future DM.

The inverse association between DM and AAA risk seems to relate mainly to hyperglycemia. Hyperglycemia might decrease AAA occurrence, as it is associated with increased collagen synthesis, elevated formation of advanced glycation end products, and decreased matrix metalloproteinases, which all may result in increased arterial stiffness through promoting cross-links between proteins such as elastin and collagen in the vessel wall, and smooth muscle cell proliferation (23). Insulin resistance, leading initially to elevated insulin concentrations, precedes the insulinopenia of frank DM. Higher insulin is associated with elevated plasminogen activator inhibitor-1 (24), which might reduce AAA 
risk through inhibiting matrix metalloproteinases production (23). Yet, in the present population-based study, elevated insulin was not associated with AAA risk.

Since individuals with DM might have been more likely to have competing risks than those without DM, and thus, we suspected this may be another explanation for the inverse association between DM and AAA risk. However, we adjusted for competing risks of death from underlying causes other than AAA, and found the inverse association was still observed.

MetS is a precursor to DM in many people. Nevertheless, MetS was associated with increased risk of AAA, because with an increasing number of non-glucose MetS components, AAA risk was higher. Although the MetS is associated with increased risk of several cardiovascular diseases (24-27), this is the first study to report a positive association between the MetS and AAA.

Several limitations of our study need to be mentioned. Firstly, our assessment of clinical AAA was only due to hospital and death $\operatorname{ICD}$ codes $(8,16)$. Although we did not directly validate $I C D$ codes for AAA, the AAA codes seem quite specific, but also insensitive for capturing AAA events. Thus, we might have missed AAA events, and certainly those with asymptomatic disease. Particularly, AAA events among those with DM might have been underestimated because hyperglycemia may result in increased arterial stiffness, leading to asymptomatic AAA. Secondly, we excluded the few participants with a history of AAA repair at baseline but did not perform a baseline ultrasound screening for AAA. Thus, some participants with asymptomatic AAA at baseline might have been included in this study. However, since our participants were 45 to 65 years old at baseline, we assume that the number of prevalent AAAs should have been low. Thirdly, we measured AAA risk factors only at baseline. Thus, we cannot negate the possibility of misclassification during the follow-up. Fourthly, we cannot negate the possibility of residual confounding due to unmeasured factors as in most observational studies, neither. Fifthly, our participants were only whites and African American, and the present findings may not be applicable to the other races such as Asian people. Thus, future research for those populations will be needed.

\section{CONCLUSIONS}

In this population-based cohort study, DM, fasting glucose, and plasma leptin were inversely associated with increased risk of AAA. In contrast, the MetS was associated with increased risk of AAA, due to the influence of the non-glucose MetS components.

\section{Supplementary Material}

Refer to Web version on PubMed Central for supplementary material.

\section{Acknowledgments}

The authors thank the staff and participants of the ARIC study for their important contributions.

\section{FUNDING SOURCES}


This work was supported by the National Heart, Lung, and Blood Institute (NHLBI) via contracts HHSN268201100005C, HHSN268201100006C, HHSN268201100007C, HHSN268201100008C, HHSN268201100009C, HHSN268201100010C, HHSN268201100011C, HHSN268201100012C, and R01 HL103695.The Nippon Foundation provided grants to support Dr. Kubota's fellowship at School of Public Health, University of Minnesota.

\section{ABBREVIATIONS}
AAA
abdominal aortic aneurysm
MetS
metabolic syndrome
DM
diabetes mellitus
ARIC Atherosclerosis Risk in Communities
ICD-9 International Classification of Diseases-9th Revision

\section{References}

1. U S. Preventive Services Task Force. Screening for abdominal aortic aneurysm: recommendation statement. Ann Intern Med. 2005; 142:198-202. [PubMed: 15684208]

2. Golledge J, Tsao PS, Dalman RL, Norman PE. Circulating markers of abdominal aortic aneurysm presence and progression. Circulation. 2008; 118:2382-92. [PubMed: 19047592]

3. Lederle FA, Johnson GR, Wilson SE, Chute EP, Hye RJ, Makaroun MS, et al. The aneurysm detection and management study screening program: validation cohort and final results. Aneurysm Detection and Management Veterans Affairs Cooperative Study Investigators. Arch Intern Med. 2000; 160:1425-30. [PubMed: 10826454]

4. Lederle FA, Larson JC, Margolis KL, Allison MA, Freiberg MS, Cochrane BB, et al. Abdominal aortic aneurysm events in the women's health initiative: cohort study. BMJ. 2008; 337:a1724. [PubMed: 18854591]

5. Bhak RH, Wininger M, Johnson GR, Lederle FA, Messina LM, Ballard DJ, et al. Factors associated with small abdominal aortic aneurysm expansion rate. JAMA Surg. 2015; 150:44-50. [PubMed: 25389641]

6. Sidloff D, Stather P, Dattani N, Bown M, Thompson J, Sayers R, et al. Aneurysm global epidemiology study: public health measures can further reduce abdominal aortic aneurysm mortality. Circulation. 2014; 129:747-53. [PubMed: 24249717]

7. Takagi H, Umemoto T. A meta-analysis of the association of obesity with abdominal aortic aneurysm presence. Int Angiol. 2015; 34:383-91. [PubMed: 24945917]

8. Tang W, Yao L, Roetker NS, Alonso A, Lutsey PL, Steenson CC, et al. Lifetime Risk and Risk Factors for Abdominal Aortic Aneurysm in a 24-Year Prospective Study: The ARIC Study (Atherosclerosis Risk in Communities). Arterioscler Thromb Vasc Biol. 2016; 36:2468-77. [PubMed: 27834688]

9. Schmidt MI, Duncan BB, Vigo A, Pankow JS, Couper D, Ballantyne CM, et al. Leptin and incident type 2 diabetes: risk or protection? Diabetologia. 2006; 49:2086-96. [PubMed: 16850292]

10. The ARIC investigators. The Atherosclerosis Risk in Communities (ARIC) Study: design and objectives. Am J Epidemiol. 1989; 129:687-702. [PubMed: 2646917]

11. Kubota Y, Heiss G, MacLehose RF, Roetker NS, Folsom AR. Educational Attainment and Lifetime Risk of Cardiovascular Disease: the Atherosclerosis Risk in Communities Study. JAMA Internal Medicine. 2017; Epub ahead of print. doi: 10.1001/jamainternmed.2017.1877

12. Dearborn JL, Schneider AL, Sharrett AR, Mosley TH, Bezerra DC, Knopman DS, et al. Obesity, Insulin Resistance, and Incident Small Vessel Disease on Magnetic Resonance Imaging: Atherosclerosis Risk in Communities Study. Stroke. 2015; 46:3131-6. [PubMed: 26451022]

13. World Health Organization homepage [Internet]. Switzerland: [updated Jun, 2016; cited July 23, 2017]. Available from: http://www.who.int/mediacentre/factsheets/fs311/en/ 
14. Salomaa V, Riley W, Kark JD, Nardo C, Folsom AR. Non-insulin-dependent diabetes mellitus and fasting glucose and insulin concentrations are associated with arterial stiffness indexes. The ARIC Study. Atherosclerosis Risk in Communities Study. Circulation. 1995; 91:1432-43. [PubMed: 7867184]

15. Folsom AR, Nieto FJ, McGovern PG, Tsai MY, Malinow MR, Eckfeldt JH, et al. Prospective study of coronary heart disease incidence in relation to fasting total homocysteine, related genetic polymorphisms, and B vitamins: the Atherosclerosis Risk in Communities (ARIC) study. Circulation. 1998; 98:204-10. [PubMed: 9697819]

16. Folsom AR, Yao L, Alonso A, Lutsey PL, Missov E, Lederle FA, et al. Circulating Biomarkers and Abdominal Aortic Aneurysm Incidence: The Atherosclerosis Risk in Communities (ARIC) Study. Circulation. 2015; 132:578-85. [PubMed: 26085454]

17. Kubota Y, London SJ, Cushman M, Chamberlain AM, Rosamond WD, Heckbert SR, et al. Lung function, respiratory symptoms and venous thromboembolism risk: the Atherosclerosis Risk in Communities Study. J Thromb Haemost. 2016; 14:2394-401. [PubMed: 27696765]

18. Enriori PJ, Evans AE, Sinnayah P, Cowley MA. Leptin resistance and obesity. Obesity (Silver Spring). 2006; 14(Suppl 5):254S-8S. [PubMed: 17021377]

19. Schmidt MI, Duncan BB, Vigo A, Pankow JS, Couper D, Ballantyne CM, et al. Leptin and incident type 2 diabetes: risk or protection? Diabetologia. 2006; 49:2086-96. [PubMed: 16850292]

20. McNeely MJ, Boyko EJ, Weigle DS, Shofer JB, Chessler SD, Leonnetti DL, et al. Association between baseline plasma leptin levels and subsequent development of diabetes in Japanese Americans. Diabetes Care. 1999; 22:65-70. [PubMed: 10333905]

21. Boyko EJ, de Courten M, Zimmet PZ, Chitson P, Tuomilehto J, Alberti KG. Features of the metabolic syndrome predict higher risk of diabetes and impaired glucose tolerance: a prospective study in Mauritius. Diabetes Care. 2000; 23:1242-8. [PubMed: 10977013]

22. Franks PW, Brage S, Luan J, Ekelund U, Rahman M, Farooqi IS, et al. Leptin predicts a worsening of the features of the metabolic syndrome independently of obesity. Obes Res. 2005; 13:1476-84. [PubMed: 16129731]

23. De Rango P, Farchioni L, Fiorucci B, Lenti M. Diabetes and abdominal aortic aneurysms. Eur J Vasc Endovasc Surg. 2014; 47:243-61. [PubMed: 24447529]

24. Festa A, D'Agostino R Jr, Mykkänen L, Tracy RP, Zaccaro DJ, Hales CN, et al. Relative contribution of insulin and its precursors to fibrinogen and PAI-1 in a large population with different states of glucose tolerance. The Insulin Resistance Atherosclerosis Study (IRAS). Arterioscler Thromb Vasc Biol. 1999; 19:562-8. [PubMed: 10073958]

25. Sattar N, Gaw A, Scherbakova O, Ford I, O'Reilly DS, Haffner SM, et al. Metabolic syndrome with and without C-reactive protein as a predictor of coronary heart disease and diabetes in the West of Scotland Coronary Prevention Study. Circulation. 2003; 108:414-9. [PubMed: 12860911]

26. Wang J, Ruotsalainen S, Moilanen L, Lepistö P, Laakso M, Kuusisto J. The metabolic syndrome predicts incident stroke: a 14-year follow-up study in elderly people in Finland. Stroke. 2008; 39:1078-83. [PubMed: 18323501]

27. Conen D, Rexrode KM, Creager MA, Ridker PM, Pradhan AD. Metabolic syndrome, inflammation, and risk of symptomatic peripheral artery disease in women: a prospective study. Circulation. 2009; 120:1041-7. [PubMed: 19738135] 


\section{Table 1}

Baseline Characteristics of Participants According to Diabetes Status (n=13,763), ARIC, 1987-1989.

\begin{tabular}{lcc}
\hline & \multicolumn{2}{c}{ Diabetes mellitus } \\
\cline { 2 - 3 } & No & Yes \\
\hline Participants, $\mathrm{n}$ & 12,376 & 1,387 \\
Age, $\mathrm{y}$ & $54.0 \pm 0.05$ & $55.9 \pm 0.15$ \\
Female, $\%$ & 55.8 & 54.1 \\
African American, \% & 23.0 & 39.2 \\
Height, cm & $168.5 \pm 0.08$ & $168.3 \pm 0.25$ \\
Current smoker, \% & 25.6 & 22.5 \\
Pack-years of smoking & $15.7 \pm 0.2$ & $17.1 \pm 0.6$ \\
Hypertension, $\%$ & 30.7 & 58.3 \\
HDL cholesterol, mmol/L & $1.4 \pm 0.004$ & $1.2 \pm 0.012$ \\
LDL cholesterol, mmol/L & $3.5 \pm 0.009$ & $3.7 \pm 0.027$ \\
Peripheral artery disease, $\%$ & 2.3 & 4.2 \\
Medication use for diabetes, $\%$ & 0 & 37.8 \\
Diabetes-related factors & & \\
Metabolic syndrome, \% & 30.8 & 77.9 \\
Fasting glucose, mg/dL & $98.6 \pm 0.2$ & $172.3 \pm 0.6$ \\
Fasting insulin, pmol/L & $77.2 \pm 1.2$ & $206.3 \pm 3.6$ \\
Leptin, ng/mL ${ }^{*}$ & $11.3 \pm 0.6$ & $14.3 \pm 1.9$ \\
\hline & & \\
\hline
\end{tabular}

Values are mean \pm standard error for continuous variables and $\%$ for categorical variables.

* Available only for a cohort random sample $(\mathrm{n}=701)$. 


\section{Table 2}

Hazard Ratios (HRs) and 95\% Confidence Intervals (CIs) for Incident, Clinical Abdominal Aortic Aneurysm According to Plasma Diabetes-related Biomarkers (n=13,763), ARIC, 1987-2013.

\begin{tabular}{|c|c|}
\hline & $\log _{2}$ (fasting glucose) \\
\hline No. at risk & 13,763 \\
\hline Person-years & 275,054 \\
\hline Cases & 518 \\
\hline Model 1 & $0.72(0.50-1.05)$ \\
\hline Model 2 & $0.54(0.36-0.80)$ \\
\hline \multirow[t]{2}{*}{ Model 2 for individuals without diabetes } & $0.76(0.38-1.50)$ \\
\hline & $\log _{2}$ (fasting insulin) \\
\hline No. at risk & 13,763 \\
\hline Person-years & 275,054 \\
\hline Cases & 518 \\
\hline Model 1 & $1.11(1.02-1.21)$ \\
\hline Model 2 & $1.04(0.94-1.15)$ \\
\hline \multirow[t]{2}{*}{ Model 2 for individuals without diabetes } & $0.76(0.38-1.50)$ \\
\hline & $\log _{2}(\text { leptin })^{*}$ \\
\hline No. at risk & 701 \\
\hline Person-years & 14,199 \\
\hline Cases & 37 \\
\hline Model 1 & $0.72(0.64-0.82)$ \\
\hline Model 2 & $0.83(0.71-0.98)$ \\
\hline Model 2 for individuals without diabetes & $0.83(0.70-0.98)$ \\
\hline
\end{tabular}

Model 1: Adjusted for age, sex, and race/ARIC field center

Model 2: Adjusted for Model 1 + adjusted for smoking status, pack-years of smoking, height, hypertension, HDL and LDL cholesterol, and peripheral artery disease.

Only available for the cohort random sample $(\mathrm{n}=701)$. 


\section{Table 3}

Hazard Ratios and 95\% Confidence Intervals for Abdominal Aortic Aneurysm According to Metabolic Syndrome (n=13,763), 1987-2013, ARIC.

\begin{tabular}{lcc}
\hline & \multicolumn{2}{c}{ Metabolic syndrome } \\
\cline { 2 - 3 } & No & Yes \\
\hline No. at risk & 8,871 & 4,892 \\
Person-years & 182,446 & 92,608 \\
Cases & 295 & 223 \\
Model 1 & 1 & $1.29(1.08-1.53)$ \\
Model 2 & 1 & $1.24(1.04-1.48)$ \\
\hline
\end{tabular}

Model 1: Adjusted for age, sex, and race/ARIC field center.

Model 2: Adjusted for Model 1 + adjusted for smoking status, pack-years of smoking, height, LDL cholesterol, and peripheral artery disease. 


\section{Table 4}

Hazard Ratios and 95\% Confidence Intervals for Incident, Clinical Abdominal Aortic Aneurysm According to Number of Metabolic Syndrome Components in Participants with or without Diabetes Mellitus, ARIC, 1987$2011(n=13,763)$.

\begin{tabular}{|c|c|c|c|c|}
\hline & \multicolumn{4}{|c|}{ Number of metabolic syndrome components other than abnormal glucose metabolism } \\
\hline & 0-1 & 2 & 3 & 4 \\
\hline \multicolumn{5}{|c|}{ Without diabetes mellitus } \\
\hline Participants, $\mathrm{n}$ & 6.928 & 3,285 & 1,633 & 530 \\
\hline Person-years & 143,625 & 65,690 & 31,778 & 10,051 \\
\hline Cases, $\mathrm{n}$ & 225 & 125 & 95 & 40 \\
\hline Model 1 & 1 & $1.16(0.93-1.44)$ & $1.61(1.27-2.05)$ & $1.99(1.42-2.79)$ \\
\hline Model 2 & 1 & $1.10(0.89-1.37)$ & $1.47(1.16-1.87)$ & $1.89(1.35-2.65)$ \\
\hline \multicolumn{5}{|c|}{ With diabetes mellitus } \\
\hline Participants, $\mathrm{n}$ & 293 & 455 & 411 & 228 \\
\hline Person-years & 5,487 & 8,120 & 6,697 & 3,606 \\
\hline Cases, $\mathrm{n}$ & 4 & 6 & 16 & 7 \\
\hline Model 1 & $0.38(0.14-1.02)$ & $0.46(0.20-1.03)$ & $1.33(0.80-2.21)$ & $1.09(0.51-2.31)$ \\
\hline Model 2 & $0.34(0.13-0.92)$ & $0.51(0.23-1.15)$ & $1.21(0.73-2.01)$ & $1.20(0.56-2.55)$ \\
\hline
\end{tabular}

Model 1: Adjusted for age, sex, and race/ARIC field center.

Model 2: Adjusted for Model $1+$ height, smoking status, pack-years of smoking, LDL cholesterol and peripheral artery disease. 